\title{
On a new species of Anagrus (Hymenoptera: Mymaridae) A note on the availability of the name Anagrus nepetellae Viggiani \& Nugnes
}

\author{
G. Viggiani, ${ }^{1}$ F. Nugnes ${ }^{2}$ \\ ${ }^{1}$ Laboratorio di Lotta Biologica, Dipartimento di Agraria, Università degli Studi di Napoli "Federico II"; ${ }^{2}$ CNR, Institute \\ for Sustainable Plant Protection, SS of Portici (NA), Italy
}

\begin{abstract}
The depository institutions of the type material of Anagrus nepetellae Viggiani and Nugnes sp. n. are indicated.
\end{abstract}

\section{Short paper}

In a previous paper (Nugnes et al., 2017) Anagrus nepetellae (Hymenoptera: Mymaridae) was described as a new species, but the collection where the holotype was deposited was omitted rendering the new name unavailable (see ICZN, 1999. International Code of Zoological Nomenclature (fourth edition) Article 16.4.2). To correct this omission we state that the holotype (ITALY. Potenza. Rivello. ANEP-001, Female 22/XII/2014 - laboratory emergence: 12/I/2015 - ex egg of Eupteryx zelleri on Clinopodium nepeta, G. Viggiani col.) of this new species named Anagrus nepetellae Viggiani and Nugnes sp. $n$. is deposited in the entomological collection (ex DEZA) of the Dipartimento di Agraria, Università degli Studi di Napoli Federico II, Portici, Napoli, Italy.

\footnotetext{
Correspondence: Gennaro Viggiani, Laboratorio di Lotta biologica Dipartimento di Agraria, Università degli Studi di Napoli "Federico II", Via Università, 133, Portici, Italia.

Tel.: + 39.081.2539003 - Fax: +39.081. 7755872.

E-mail: genviggi@unina.it

Key words: nepetellae, calamint, leafhopper, parasitoid.

Contributions: the authors contributed equally.

Conflict of interest: the authors declare no potential conflict of interest.

Funding: none.

Received for publication: 19 March 2018.

Accepted for publication: 30 April 2018.

CCopyright G. Viggiani and F. Nugnes, 2018

Licensee PAGEPress, Italy

Journal of Entomological and Acarological Research 2018; 50:7428

doi:10.4081/jear.2018.7428

This article is distributed under the terms of the Creative Commons Attribution Noncommercial License (by-nc 4.0) which permits any noncommercial use, distribution, and reproduction in any medium, provided the original author(s) and source are credited.
}

**** female paratypes and $* * *$ male paratypes, as reported in the supplementary material (file 2) of the paper Nugnes et al. (2017), are deposited in the following institutions:

Dipartimento di Agraria, Università degli Studi di Napoli Federico II, Portici, Napoli, Italy (DEZA); Natural History Museum, London, England, UK (NHM); University of California, Riverside, California, USA (UCR); Canadian National Collection of Insects, Ottawa, Ontario, Canada (CNCI); National Museum of Natural History, Washington, D.C., USA (USNM).

The new species Anagrus nepetellae Viggiani and Nugnes has been registered in Zoobank with the following registration code: DFCD7654-5AA5-4E4F-AE48-24377BAACEFE.

\section{References}

ICZN, 1999 - Internatioanl Code of Zoological Nomenclature (fourth edition) xxix $+306 \mathrm{pp}$. Internatioanl Trust for Zoological Nomenclature, London, UK.

NUGNES F., BERNARDO U., VIGGIANI G., 2017 - An integrative approach to species discrimination in the Anagrus atomus group sensu stricto (Hymenoptera: Mymaridae), with a description of a new species. Syst. Biodiv. 15: 582-99. 